\title{
Commodity markets, price limiters and speculative price dynamics
}

\author{
Xue-Zhong $\mathrm{He}^{\mathrm{a}}$, Frank H. Westerhoff ${ }^{\mathrm{b}, *}$ \\ ${ }^{a}$ School of Finance and Economics, University of Technology, Sydney, Australia \\ ${ }^{\mathrm{b}}$ Department of Economics, University of Osnabrueck, Rolandstrasse 8, D-49069 Osnabrueck, Germany
}

Received 18 December 2003; accepted 16 September 2004

\begin{abstract}
We develop a behavioral commodity market model with consumers, producers and heterogeneous speculators to characterize the nature of commodity price fluctuations and to explore the effectiveness of price stabilization schemes. Within our model, we analyze how nonlinear interactions between market participants can create either bull or bear markets, or irregular price fluctuations between bull and bear markets through a (global) homoclinic bifurcation. Both the imposition of a bottoming price level (to support producers) or a topping price level (to protect consumers) can eliminate such homoclinic bifurcations and hence reduce market price volatility. However, simple policy rules, such as price limiters, may have unexpected consequences in a complex environment: a minimum price level decreases the average price while a maximum price limit increases the average price. In addition, price limiters influence the price dynamics in an intricate way and may cause volatility clustering. (C) 2004 Elsevier B.V. All rights reserved.
\end{abstract}

JEL classification: D84; G18; Q11

Keywords: Commodity markets; Price stabilization; Simple limiters; Technical and fundamental analysis; Bifurcation analysis; Chaos control

\footnotetext{
${ }^{*}$ Corresponding author. Tel.: + 4905419692743 ; fax: + 49054196912742 .

E-mail address: fwesterho@oec.uni-osnabrueck.de (F.H. Westerhoff).
} 


\section{Introduction}

Commodity prices are, by any standard, extremely volatile. After inspecting 13 primary commodities over the period 1900-1987 (deflated annual data), Deaton and Laroque (1992) found price variation coefficients, defined as the standard deviation over the mean, ranging from 0.17 (bananas) to 0.60 (sugar). In addition, one often observes dramatic boom and bust episodes. For instance, the decline in prices from the highest level reached in the period from 1974 to August 1975 was 67 percent for sugar, 58 percent for sisal, more than 40 percent for cotton and rubber, and more than 25 percent for cocoa and jute (Newbery and Stiglitz, 1981). In a recent study, Osborne (2003) reported that in Ethiopia the price of maize has more than doubled three times over the last 15 years.

Not only many developing countries, but also the United States and the European Union, have thus experimented with some form of commodity price stabilization scheme in the past. In particular, attempts have been made to stabilize agricultural commodity markets by means of a commodity buffer stock scheme. The idea of such schemes is to put a certain amount of output into storage in years in which there is a good harvest, thus increasing the price from what it would have been, and to sell output from the storage in years in which there is a small harvest, thus reducing the price from what it would have been. Another prominent example is the oil market. Following the oil crises in the 1970s, many countries built up huge oil reserves in order to influence the market.

Demand and supply schedules, storage and fully rational speculators are the key elements in neo-classical commodity market models (Waugh, 1944; Brennan, 1958; Williams and Wright, 1991; Deaton and Laroque, 1992, 1996; Chambers and Bailey, 1996; Osborne, 2003). While these models undoubtedly capture some important aspects of commodity markets, their ability to mimic features such as bubbles and crashes is, however, limited. Supporters of these models - in which the markets are efficient by nature - judge commodity price stabilization schemes as unlikely to have a significant beneficial effect (Newbery and Stiglitz, 1981).

Contrary to the efficient market hypothesis, however, there is not only widespread populist feeling that speculators are a major cause of price instability, but also theoretical papers have started to explore this aspect. The chartist-fundamentalist approach, developed in the last decade, offers a new and promising alternative behavioral perspective of financial market dynamics. The main feature of this approach is that interactions between heterogeneous agents, so-called chartists and fundamentalists, may generate an endogenous nonlinear law of motion of asset prices. In Day and Huang (1990), Chiarella (1992) and Farmer and Joshi (2002), the nonlinearity originates from nonlinear technical and fundamental trading rules whereas in Kirman (1991), Brock and Hommes (1998) and Lux and Marchesi (2000), the nonlinearity is caused by the agents switching between a given set of predictors. More recent refinements and applications include Chiarella and $\mathrm{He}$ (2001), Chiarella et al. (2002) and Westerhoff (2003). Since these models have demonstrated their ability to match the stylized facts of financial markets quite well one may conclude that this framework is suitable to conduct some policy evaluation experiments. 
This paper aims at developing a commodity market model along the lines of the chartist-fundamentalist approach to characterize price fluctuations and to unravel the potential effects of price limiters. Its main ingredients are as follows: For simplicity, demand and supply schedules are expressed in a reduced log-linear form. Fundamental to the model is the behavior of the speculators who switch between technical and fundamental trading rules to determine their positions in the market. Prices adjust via a log-linear price impact function: Excess supply (demand) decreases (increases) the price. Our model shows that: (i) the chartists are a source of market instability, as commonly believed, (ii) weak reaction of the speculators (either the fundamentalists or the chartists) can push the market to be either a bull or a bear market (through pitchfork bifurcations); and (iii) strong reaction of the speculators causes market prices to switch irregularly between bull and bear markets (through homoclinic bifurcations). Since prices fluctuate in a complex way between bull and bear markets, the model is capable of replicating some features of commodity price motion.

The paper then focuses on the impact of simple price limiters as a potential stabilizing mechanism to reduce price fluctuations. Both theoretical analysis and numerical simulations reveal that if a central authority guarantees a minimum price, e.g. to support the producers, volatility declines. Although the price is backed up from below, the average price of the commodity surprisingly decreases, too. Setting up an upper price limit, e.g. to protect consumers from excessive prices, again yields a drop in price variability. However, the average price the consumers have to pay increases. At least at first sight, this result appears to be counterintuitive and should give policy-makers a warning. Simple measures to control prices may have surprising consequences in a nonlinear world.

This puzzling outcome is caused by a dynamic lock-in effect. Consider the case of a crash without a price limiter mechanism. Within our model, a bull market turns into a bear market after the price has crossed a critical upper level. A central authority that intervenes successfully against high prices obviously destroys the necessary condition for such a regime shift. As a result, the average price is higher than without an upper price restriction. Moreover, since the price fluctuates at a high level, it reaches the upper price boundary repeatedly so that the buffer stock is likely to run empty rather quickly. We show that one way to counter this problem is to alternate temporarily between an upper and a lower price boundary. The price volatility then decreases, yet the market remains distorted. However, on-off switching of the stabilization mechanism as well as changing the level of price limiters interferes with the price discovery process and may cause severe bubbles and crashes or volatility clustering.

As it turns out, price limiters as applied in our model are identical to a recently developed chaos control method. The development of chaos control algorithms was initiated by Ott et al. (1990) (henceforth OGY). Other popular suggestions include, for instance, the delayed feedback control method of Pyragas (1992) or the constant feedback method of Parthasarathy and Sinha (1995). The OGY control scheme and its descendants have been applied in various fields such as mechanics, electronics or chemistry. Economic applications include Kopel (1997), Kaas (1998) or Westerhoff 
and Wieland (2004). The feasibility of using chaos controllers in reality depends on the complexity and efficiency of the control algorithm. The chaos control process requires measurement of the system's state, generation of a control signal, and the application of the control signal to an accessible system parameter. For instance, the original OGY control scheme requires knowledge of the map and its fixed point. While such information may be identified from observations in natural science applications, chaos control in an economic context is often seen as rather critical.

However, Corron et al. (2000) present experimental evidence that chaos control can be accomplished using simple limiters and argue that chaos control can be practically applied to a much wider array of important problems than thought possible until recently. This method, which has been analytically and numerically explored by Wagner and Stoop (2000) and Stoop and Wagner (2003), simply restricts the phase space that can be explored. Suppose that a variable fluctuates between $0<x<1$. A limiter from below resets all values $x<h$ to $h$. As a result, the new system may replace previously chaotic behavior with periodic behavior. One advantage of the limiter method is that it does not add complexity to the system by increasing the size of the system's state space. Another advantage is that stabilization may already be achieved by infrequent interventions. As far as we are aware, this paper contains the first economic study of limiters. And indeed, the method is able to decrease price fluctuations quite easily, yet with the (economic) disadvantage of a lock-in effect as stressed above.

The remainder of this paper is organized as follows. Section 2 presents a simple commodity market model with heterogeneous interacting agents and, by using stability and bifurcation analysis, Section 3 examines the price dynamics of the model without price limiter mechanisms. In Section 4, we discuss the consequences of single-price limiters for the price dynamics, and in Section 5, we introduce conditional price limiters. The final section concludes the paper.

\section{The model}

As reported in the introduction, commodity markets are extremely volatile and regularly display severe bubbles and crashes. Such price dynamics may, of course, be triggered by demand and supply shocks. Moreover, cobweb models show that complicated price motion may also occur due to nonlinearities (Hommes, 1994; Brock and Hommes, 1997; Goeree and Hommes, 2000; Chiarella and He, 2003). However, there may exist an additional source for market instability. Note that many commodities are traded at stock exchanges (e.g. at the Chicago Mercantile Exchange). As is well known, speculators take large positions in commodity markets. For empirical accounts on the behavior of speculators in these markets, see, e.g. Smidt (1965), Draper (1985), Canoles et al. (1998), Sanders et al. (2000) or Weiner (2002). Surprisingly, this aspect has received only scant attention so far. In this paper, we thus develop a stylized commodity market model with heterogeneous speculators to study the implications of some popular price stabilization schemes. To understand the workings of these mechanisms, we strive to design a model that is as 
simple as possible. Since our focus is on the activity of speculators, we describe the demand and supply decisions of consumers and producers, which have already been studied in detail, in a reduced form.

Moreover, we can apply well-established building blocks used in the literature on chartist and fundamentalist interactions. That is, we consider a market with three types of agents: consumers, producers and speculators. Speculators are heterogeneous in the sense that they are aware of both technical and fundamental trading strategies, and, at the beginning of each trading period, they choose one of the two strategies as their trading strategy for the trading period. Their behavior may be regarded as boundedly rational since the selection of a strategy depends on market circumstances.

Following Farmer and Joshi (2002), we assume that the price adjustment on the commodity market may be approximated by a log-linear price impact function. Hence, the $\log$ of the price $S$ of the commodity at time $t+1$ is

$$
S_{t+1}=S_{t}+a\left(D_{t}^{\mathrm{M}}+W_{t}^{\mathrm{C}} D_{t}^{\mathrm{C}}+W_{t}^{\mathrm{F}} D_{t}^{\mathrm{F}}\right),
$$

where $a$ is a positive scaling coefficient to calibrate the price adjustment speed, $D_{t}^{\mathrm{M}}$, $D_{t}^{\mathrm{C}}$ and $D_{t}^{\mathrm{F}}$ stand for the excess demand of the real economy, the chartists and the fundamentalists, respectively, at time $t$. The weight of the chartists at time $t$ is given as $W_{t}^{\mathrm{C}}$, whereas the weight of the fundamentalists is given as $W_{t}^{\mathrm{F}}$. According to (1), the (log of the) commodity price increases when there is an excess demand, and vice versa.

We use a reduced form to describe the demand and supply decisions of the real economy. Suppose that the long-run demand and supply schedules of consumers and producers are log-linear. Then the excess demand may be expressed as

$$
D_{t}^{\mathrm{M}}=m\left(F-S_{t}\right),
$$

where $m$ reflects the slopes of the demand and supply curves. The excess demand of the real economy is zero when the log of the price is equal to the log of the long-run equilibrium price $F$, also called the fundamental value. We assume that the structure of the economy is stable, i.e. there are no (permanent) demand and supply shocks. As a result, $F$ is constant over time and common knowledge. Note that in the absence of speculators $\left(W_{t}^{\mathrm{C}}=W_{t}^{\mathrm{F}}=0\right)$, the law of motion of the commodity's log price has a unique fixed point at $S_{t}=F$, which is stable for $0<a m<2$. Such a state is obviously efficient.

Speculators are familiar with both technical and fundamental analysis. Indeed, the use of destabilizing trend extrapolation and stabilizing mean reversion trading strategies has been confirmed in many empirical studies (Smidt, 1965; Draper, 1985; Canoles et al., 1998; Sanders et al., 2000; Weiner 2002) as well as in laboratory experiments (Smith, 1991; Sonnemans et al., 2004). To model the excess demand generated by technical analysis we adopt a formulation of Day and Huang (1990).

$$
D_{t}^{\mathrm{C}}=b\left(S_{t}-F\right),
$$

where $b$ is a positive reaction coefficient. So-called chartists typically believe in bear and bull markets. As long as the price is above its fundamental value, chartists 
regard the market as bullish. Since a further price increase is expected, chartists tend to buy the commodity. However, if the price drops below its fundamental value then the chartists become pessimistic. In a bear market, chartists sell the commodity. Since changes in excess demand are positively correlated with changes in price, (3) is - in a broader sense - consistent with positive feedback trading.

Fundamental analysis presumes that prices revert toward their fundamental value. If the price is below (above) its equilibrium value, higher (lower) prices are expected and fundamental analysis favors buying (selling) the commodity. The excess demand generated by fundamental analysis may be formalized as

$$
D_{t}^{\mathrm{F}}=c\left(F-S_{t}\right) .
$$

The reaction coefficient $c$ is positive.

The switching mechanism is based on an argument put forward by Hommes (2001). Speculators try to exploit bull and bear market situations. However, the more the price deviates from its fundamental value, the greater the speculators perceive the risk that the bull or bear market might collapse. As a result, an increasing number of speculators opt for fundamental trading strategies. The market share of speculators who follow technical analysis may thus be defined as

$$
W_{t}^{\mathrm{C}}=\frac{1}{1+d\left(F-S_{t}\right)^{2}} .
$$

The higher the switching parameter $d>0$, the faster speculators switch to fundamental analysis as the mispricing increases. The weight of fundamentalists is $W_{t}^{\mathrm{F}}=1-W_{t}^{\mathrm{C}}$.

The recurrence relation that determines the dynamics of the model is obtained by combining (1)-(5)

$$
S_{t+1}=S_{t}+a\left[m\left(F-S_{t}\right)-b \frac{\left(F-S_{t}\right)}{1+d\left(F-S_{t}\right)^{2}}+c \frac{d\left(F-S_{t}\right)^{3}}{1+d\left(F-S_{t}\right)^{2}}\right],
$$

which is a one-dimensional nonlinear map. ${ }^{1}$

\section{Price dynamics without price limiters}

Model (6) can be written as

$$
S_{t+1}=f\left(S_{t}\right)
$$

\footnotetext{
${ }^{1}$ Our model is inspired by the seminal work of Day and Huang (1990), who derive a nonlinear stock market model by showing that the fundamentalists' demand function may be S-shaped. Their model is able to produce irregular fluctuations between bull and bear markets. Indeed, the dynamics of both models is caused by a cubic one-dimensional map. The cubic nature of our model basically results from the agent's switching between strategies.
} 
where

$$
f(x)=x+a(x-F)\left[-m+\frac{b-c d(F-x)^{2}}{1+d(F-x)^{2}}\right]
$$

and $a, b, c, d$ and $m$ are positive constants. Since parameter $a$ can be treated as a scaling factor, we assume $a=1$. Accordingly, we have

$$
f(x)=x+(x-F)\left[-(m+c)+\frac{b+c}{1+d(F-x)^{2}}\right] .
$$

We now consider some properties of the map, including symmetry, invertibility, existence of fixed points, their stability, bifurcations, homoclinic orbits, and routes to complicated price dynamics. The cubic nature of the one-dimensional map has been well studied and explained analytically in the literature, see, e.g. Devaney (1989), Day and Huang (1990) and more recently Dieci et al. (2001). The analysis will improve our understanding of the price dynamics when price limiters are imposed.

Symmetry property: It is easy to verify that $f(2 F-x)=2 F-f(x)$. Hence, the map $f$ is symmetric with respect to $F$. This implies that any cycle, if it exists, is either symmetric with respect to $F$ or admits a symmetric cycle.

Fixed points, local stability and bifurcation: Let $\bar{x}$ be the steady state of the map $x_{t+1}=f\left(x_{t}\right)$. Then it satisfies

$$
(\bar{x}-F) \frac{(b-m)-(c+m) d(F-\bar{x})^{2}}{1+d(F-\bar{x})^{2}}=0 .
$$

Obviously, the $\log$ of the fundamental value $\bar{x}=F$ is always a steady state. In addition, when $b>m$, there exist two non-fundamental steady states

$$
S_{ \pm}=F \pm \sqrt{\frac{b-m}{(c+m) d}}
$$

which are, of course, symmetric to $F$.

It follows from (9) that

$$
f^{\prime}(x)=1-m-c+\frac{b+c}{1+d(x-F)^{2}}\left[-1+\frac{2}{1+d(x-F)^{2}}\right] .
$$

The eigenvalue at $F$ is given by $\lambda=f^{\prime}(F)=1-m+b$. Therefore, $\lambda=1$ when $m=b$ and $\lambda=-1$ when $b=m-2$. Accordingly, $F$ is locally asymptotically stable (LAS) when either $m \leqslant 2$ and $0 \leqslant b<m$ or $m>2$ and $m-2<b<m$. For $b>m$, there exist two non-fundamental steady states $S_{ \pm}$with eigenvalues $\lambda=f^{\prime}\left(S_{ \pm}\right)=1-$ $2((m+c)(b-m) /(b+c))$. Obviously, $\lambda=1$ when $b=m$ and $\lambda=-1$ when $b=$ $(c+m(m+c)) /(m+c-1)$. Let

$$
b_{1}=m-2, \quad b_{2}=m-1 \text { and } b_{3}=\frac{c+m(m+c)}{m+c-1} .
$$

Note that $b_{1}<b_{2}<m<b_{3}$ for $m+c>1$. The analysis leads to the following results. 
Proposition 1. For model (6),

- the log of the fundamental value $F$ is always a steady state,

- there exist two additional non-fundamental steady states $S_{ \pm}$when $b>m$,

- the steady state $F$ is LAS if either $m \leqslant 2$ and $0 \leqslant b<m$ or $m>2$ and $b_{1}<b<m$ (F is unstable for $b>m$ ),

- a pitchfork bifurcation occurs at $b=m$,

- both non-fundamental steady states $S_{ \pm}$are $L A S$ if either $m+c \leqslant 1$ and $b>m$ or $m+c>1$ and $m<b<b_{3}$,

- a period-doubling bifurcation occurs at $b=b_{3}$ if $m+c>1$.

It is well known that, in the standard (linear) cobweb model, the fundamental value is stable only if the slope of demand and supply is $m<2$. Proposition 1 indicates that a limited extrapolation from the chartists is stabilizing an otherwise unstable fundamental value. In fact, $F$ is LAS when the slope of the demand and supply satisfies $m<2$ and the chartists extrapolate weakly $(b<m)$. For $m>2$, the local stability of $F$ is regained when a limited extrapolation from the chartists is allowed $(m-2<b<m)$. Note that the fundamental value can lose its stability through a pitchfork bifurcation when the chartists extrapolate strongly $(b>m)$, leading to two LAS non-fundamental steady states $S_{ \pm}$. Therefore, the extrapolation of the chartists has a double-edged effect in terms of the stability of the fundamental value: A weak (strong) extrapolation of chartists is stabilizing (destabilizing). ${ }^{2}$ In addition, the non-fundamental steady states $S_{ \pm}$increasingly deviate from $F$ as the extrapolation from the chartists increases and they lose stability through a perioddoubling bifurcation, leading to two stable cycles of period two. Further bifurcations and dynamics depend on the invertibility of the map. Note that, for $b>m$ and $m+c>1, b_{3}$ decreases as $c$ increases. In addition, $b_{3} \rightarrow m+1$ as $c \rightarrow \infty$ and $b_{3} \rightarrow m^{2} /$ $(m-1)$ as $c \rightarrow 0$.

Invertibility of the map: The map is invertible if both forward and backward iterations are uniquely defined. As regards the conditions for the map to be invertible, we have the following result (proven in Appendix A).

Proposition 2. When $m<1$, the map is invertible for $m+c \leqslant 1$ and not invertible for $m+c>1$. When $m \geqslant 1$, the map is invertible for $b \leqslant b_{2}=m-1$ and not invertible for $b>b_{2}$.

Note that Proposition 2 implies that for $m<1$, the map becomes not invertible when the fundamentalists react strongly $(m+c>1)$. For $m \geqslant 1$, the map becomes not invertible when the chartists extrapolates strongly $\left(b>b_{2}\right)$. Hence, the two types of speculators play different roles.

Trapping intervals, invariant sets and homoclinic bifurcation: Based on the above analysis it is clear that once the reaction parameters $b$ or $c$ cross a certain threshold, the map becomes not invertible. To illustrate the local and global behavior of the map, we now follow a particular bifurcation route to homoclinic bifurcation and complicated price dynamics by choosing $c=1.5, d=1, m=1, F=0$ and the

\footnotetext{
${ }^{2}$ This effect is also reported in Chiarella and He (2003) where heterogeneous learning is studied in a cobweb model.
} 
extrapolation rate of the chartists $b$ as the changing parameter. Since $m=1$ and $m+c=2.5>1$, it follows from Proposition 2 that the map is not invertible for $b>m-1=0$. The first two panels of Fig. 1 show that the log of the fundamental value $F=0$ is stable for $0<b<1$ and unstable for $b>1$. A pitchfork bifurcation occurs at $b=1$, leading to two symmetric (locally) stable non-fundamental steady states $S_{ \pm}$for $1<b<b_{3}=2.667$. Because the map is non-invertible, the global basins of each nonfundamental steady state $S_{ \pm}$is made up of infinitely many disjoint intervals. At $b=b_{3}$, two symmetric stable cycles of period two appear. As $b$ increases further, each stable two-cycle of the map undergoes a flip bifurcation, leading to a new attractive periodfour-cycle and so on. Therefore, the map undergoes a typical route of period doubling to complex price dynamics, which characterizes the price dynamics in either the bull or the bear market. At $b \approx 4.36$ a homoclinic bifurcation occurs. ${ }^{3}$

For the same set of parameters, panels (a)-(c) of Fig. 2 further illustrate the impact of the homoclinic bifurcation of the map (without price limiter). Because of the noninvertibility of the map, there exist two values $S_{\mathrm{m}}$ and $S_{\mathrm{M}}$ satisfying $S_{\mathrm{m}}<F<S_{\mathrm{M}}$ such that the map has a local minimum at $S_{\mathrm{m}}$ and a local maximum at $S_{\mathrm{M}}$. Denote $F_{\mathrm{m}}=f\left(S_{\mathrm{m}}\right)$ and $F_{\mathrm{M}}=f\left(S_{\mathrm{M}}\right)$. As illustrated for instance in Devaney (1989) (see also Day and Huang, 1990; Dieci et al., 2001 for economic applications), the homoclinic bifurcation occurs precisely when the local maximum $F_{\mathrm{M}}$ and the local minimum $F_{\mathrm{m}}$ are exactly mapped into the repelling steady state $F$, that is, $f\left(F_{\mathrm{m}}\right)=f\left(F_{\mathrm{M}}\right)=F$. This is the case for $b \approx 4.36$. Panel (a) of Fig. 1 reveals that for $b=4$, the intervals $I_{\mathrm{m}}=\left[F_{\mathrm{m}}\right.$, $\left.f\left(F_{\mathrm{m}}\right)\right]$ and $I_{\mathrm{M}}=\left[F_{\mathrm{M}}, f\left(F_{\mathrm{M}}\right)\right]$ are invariant trapping intervals satisfying $I_{\mathrm{m}} \cap I_{\mathrm{M}}=\varnothing$ and prices are locked in either the bull or the bear market. At the homoclinic bifurcation value $b \approx 4.36$ (panel (b)), the two invariant trapping intervals join together. After the homoclinic bifurcation $(b=4.5)$, the intervals $I_{\mathrm{m}}$ and $I_{\mathrm{M}}$ are no longer invariant intervals. In fact, $I_{\mathrm{m}} \cap I_{\mathrm{M}} \neq \varnothing$. Instead their union $I=I_{\mathrm{m}} \cup I_{\mathrm{M}}$ becomes the new trapping invariant interval. Consequently, the two disjoint onepiece complicated attractors merge into a single piece of complicated attractor, characterizing the irregular switching between bull and bear markets. ${ }^{4}$ Overall, the existence of a homoclinic bifurcation leads to a remarkable qualitative change in the price dynamics, routing to complicated price dynamics.

The local and global bifurcations are summarized in Fig. 3 which illustrates the local stability regions of the steady-state prices and the various bifurcation curves in parameter space $(b, c)$ for given $d=1, m=1$ and $F=0$.

\section{Simple price limiters}

Next, we study the consequences of the limiter method - as suggested by Corron et al. (2000) - on commodity price dynamics. Price limiters may easily be implemented

\footnotetext{
${ }^{3}$ The bottom two panels of Fig. 1 display bifurcation diagrams with respect to parameter $c$, i.e. the reaction coefficient of the fundamentalists. Note that similar routes to complicated dynamics occur.

${ }^{4}$ See also the first two bifurcation plots of Fig. 1 and the top panel of Fig. 4 (next section). The latter presents an example of the intricate price dynamics in the time domain.
} 

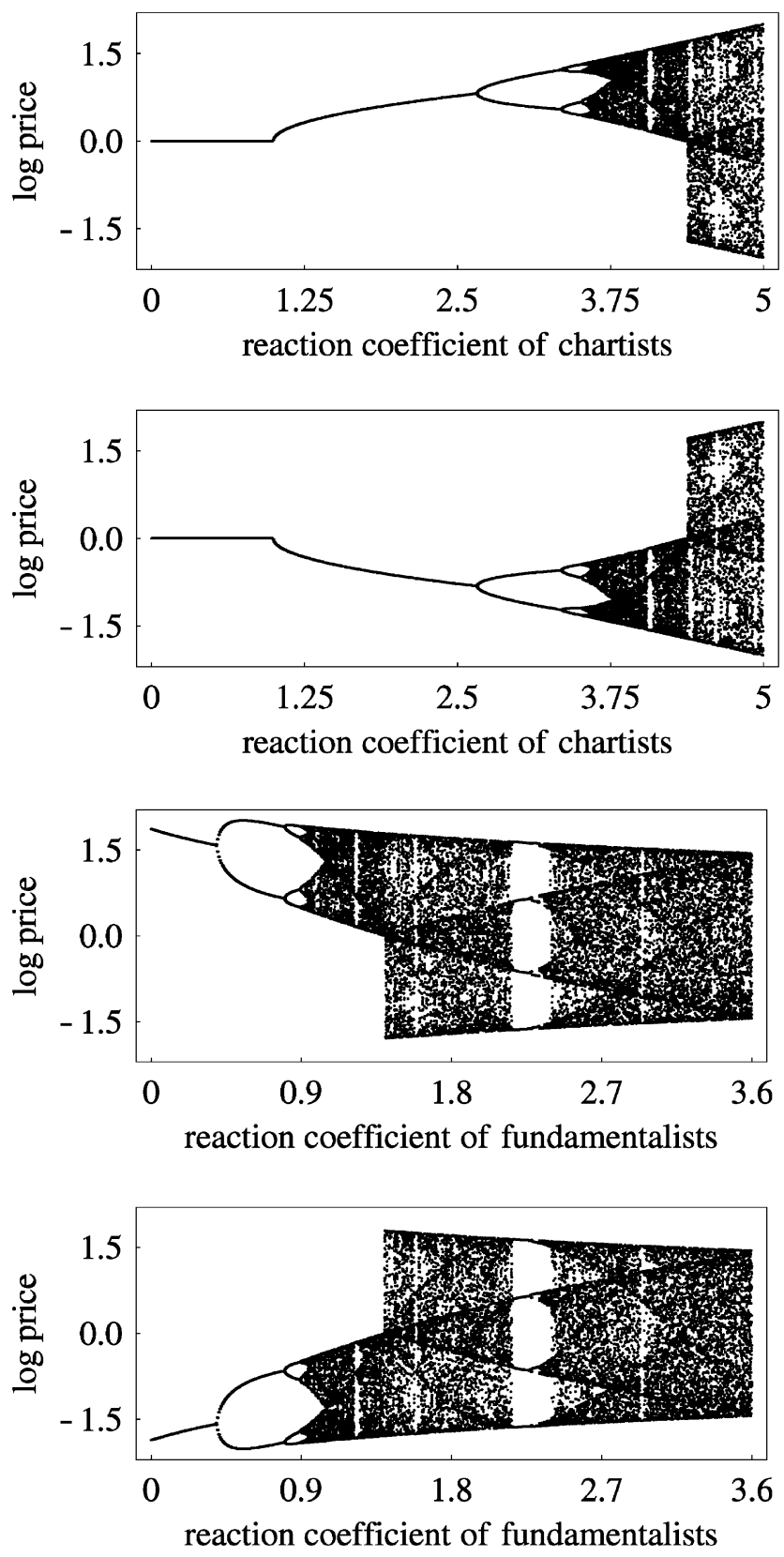

Fig. 1. Bifurcation diagrams for parameters $b$ and $c$ using different initial conditions. The parameters are increased in 500 steps as indicated on the axis. Log prices are plotted from $t=500-600$. The other parameters are $a=1, b=4.5, c=1.5, d=1, m=1$ and $F=0$. 

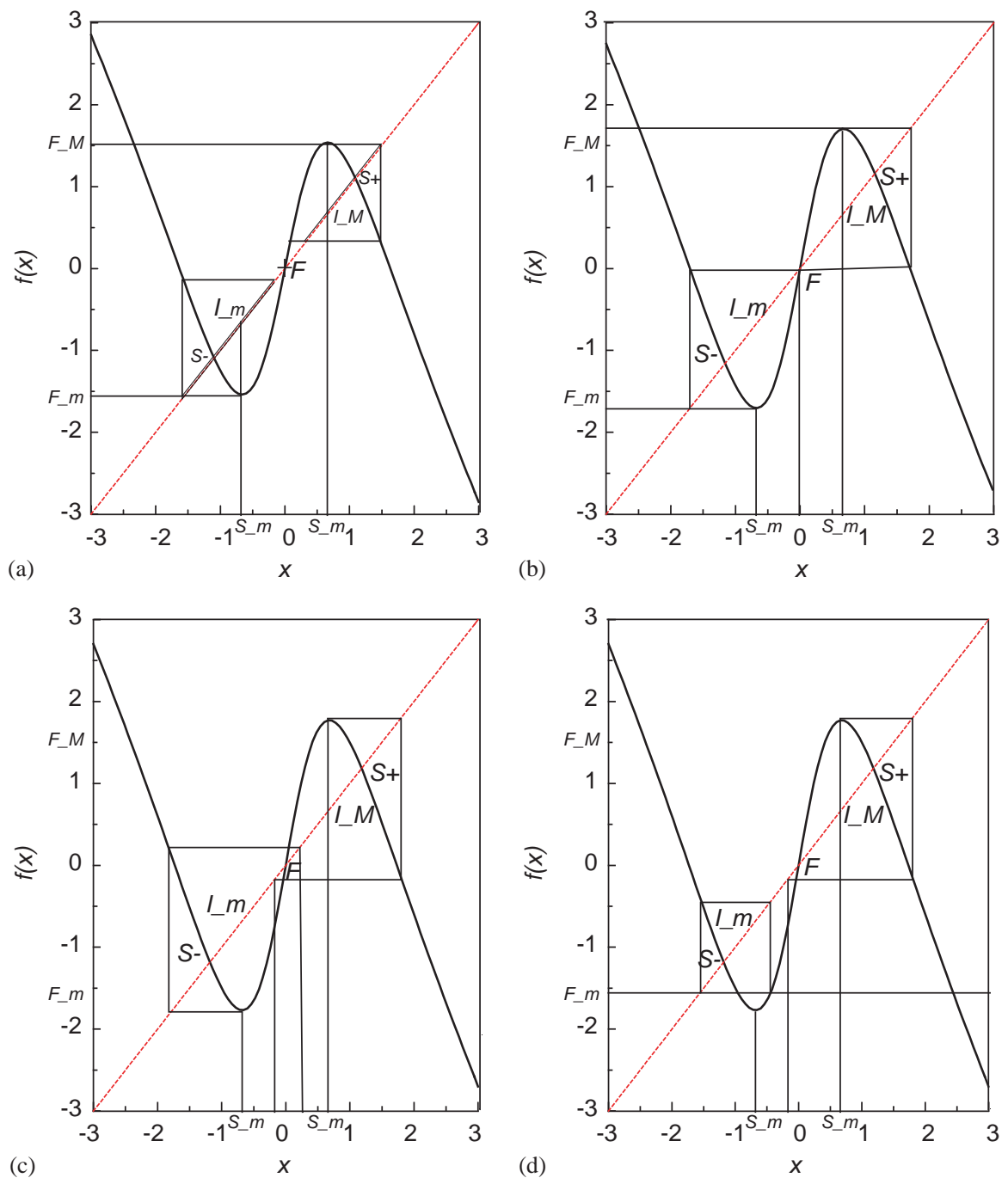

Fig. 2. Homoclinic bifurcation of the map without price limiter (a)-(c) and with price limiter (d). The other parameters are $a=1, c=1.5, d=1, m=1$ and $F=0$. (a) $b=4$, (b) $b=4.36$, (c) $b=4.5$ and (d) $b=4.5$ and $S^{\mathrm{min}}=-1.6$.

in our framework. In the case of a minimum $\log$ price $S^{\text {min }}$, (1) becomes

$$
S_{t+1}=\operatorname{Max}\left[f\left(S_{t}\right), S^{\mathrm{min}}\right],
$$

in the case of a maximum $\log$ price $S^{\max }$, the price adjustment modifies according to

$$
S_{t+1}=\operatorname{Min}\left[f\left(S_{t}\right), S^{\max }\right]
$$




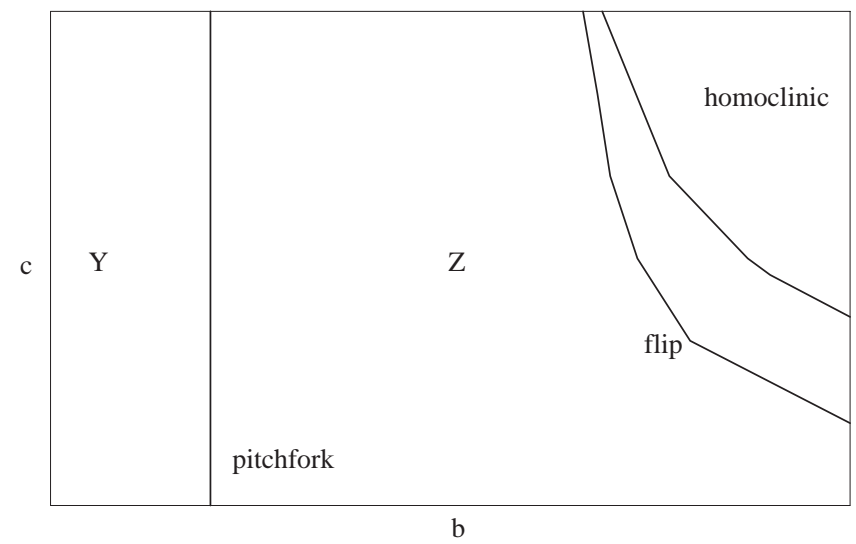

Fig. 3. Local stability region $\mathrm{Y}$ for the $\log$ of the fundamental value $F$ and $\mathrm{Z}$ for the two non-fundamental steady states $S_{ \pm}$in parameter space $(b, c)$. In addition, pitchfork, flip and homoclinic bifurcation curves. The other parameters are $a=1, d=1, m=1$ and $F=0$.

and in the case of a minimum and a maximum log price restriction, one obtains

$$
S_{t+1}=\operatorname{Min}\left[\operatorname{Max}\left[f\left(S_{t}\right), S^{\min }\right], S^{\max }\right] .
$$

In order to avoid black markets, the central authority has, of course, to intervene in the market. For instance, to prevent the price from dropping below the minimum price the central authority has to buy a fraction of the supplied commodity.

Let us start the analysis by comparing the two time series displayed in Fig. 4, where we assume $a=1, b=4.5, c=1.5, d=1, m=1$ and $F=0$. The top panel of Fig. 4 shows the evolution of the log of the commodity price without a price limiter in the time domain (200 observations). Visual inspection reveals the model's ability to produce bubbles and crashes, as observed in many commodity markets.

Concerned with the turbulent dynamics, a central authority may try to support the producers by guaranteeing them a minimum price. The bottom panel of Fig. 4 presents a simulation run in which $S^{\mathrm{min}}=-1.6$. The impact of such a price stabilization scheme may be quite dramatic. Note first that the price fluctuations appear to be much lower. And in fact, the variance of the price drops from around 1.11 (no price restriction) to 0.18 . But the average price is also affected by this policy. Now, the price always fluctuates below its fundamental value. To be precise, the average $\log$ price decreases from about 0 to -1.07 (all statistics are based on simulation runs with 10,000 observations). At least at first sight, this is surprising. The central authority aims to protect the producers from too low prices, yet the average price drops.

On first sight, this outcome may appear puzzling. However, the analysis of the previous section helps us to understand this phenomenon. Note that any interruption of the existence of the homoclinic bifurcation, such as price limiters, will rule out possible random switching between bull and bear markets and lead prices to be trapped in either the bull or the bear market. Panel (d) of Fig. 2, which is 

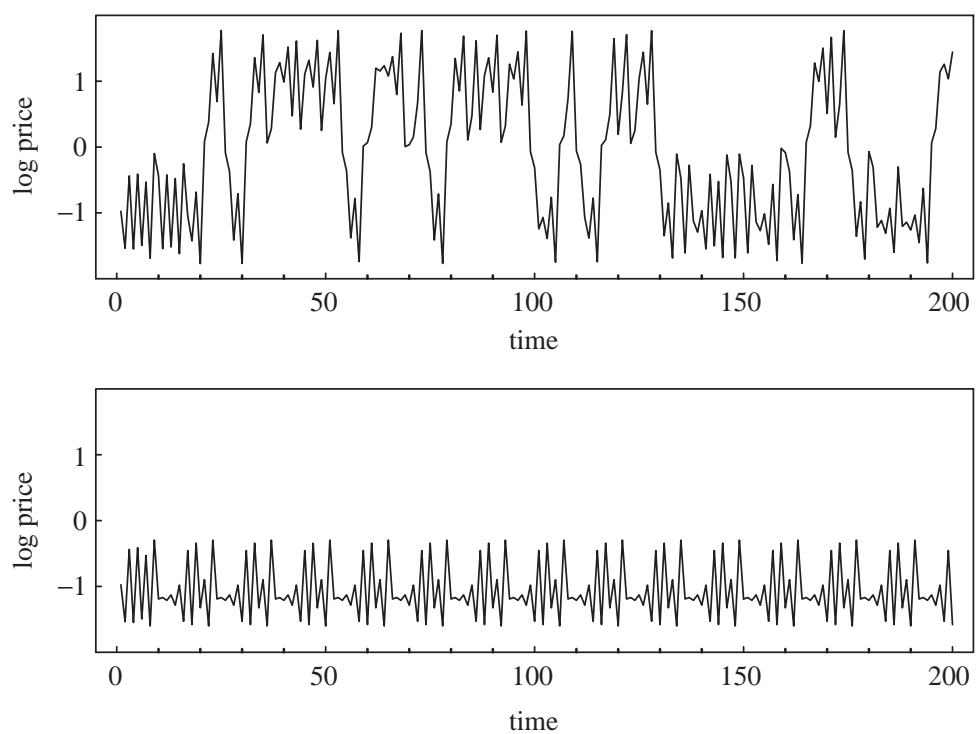

Fig. 4. The top panel shows the unrestricted evolution of the commodity's log price in the time domain. The bottom panel shows the same, but with a lower price boundary of $S^{\mathrm{min}}=-1.6$. The other parameters are $a=1, b=4.5, c=1.5, d=1, m=1$ and $F=0$.

based on the same parameter setting as panel (c), shows the impact of a price limiter on the price dynamics. Now it is clear that a change from a bear market into a bull market requires the price to drop to a rather low value. But, due to the price cap, the map in panel (d) is flat at $S^{\mathrm{min}}=-1.6$. This prevents the system from switching from a bear market to a bull market. Clearly, the price is trapped below its fundamental value.

Fig. 5 further investigates this dynamic lock-in effect. The top left panel shows a bifurcation diagram in which the minimum log price is increased in 500 steps from -2 to 0 . To allow the system to settle on its attractor, the dynamics is plotted for log prices between the periods 500 and 600 . As can be seen, the complicated behavior turns into periodic behavior as the minimum log price increases. At around $p^{\mathrm{min}}=$ -1.18 , even a fixed point emerges. The top right panel reveals symmetrical results for an upper price boundary, which decreases from 2 to 0 .

The second and third panels in Fig. 5 present how the mean and the variance of the price react to a change in the price limit. To obtain reasonable statistics, IID shocks with white noise $N(0,0.1)$ are added to the system at every time step. The limiter method indeed proves its power in stabilizing the dynamics. Already minor price restrictions may eliminate the larger part of the price variability. But the average price is simultaneously affected. Restricting the price from below (above) increases (decreases) the mean of the price in an adverse manner. Only if the restrictions are very sharp may a lower (higher) price boundary lead to an increase (decrease) in the average price. 

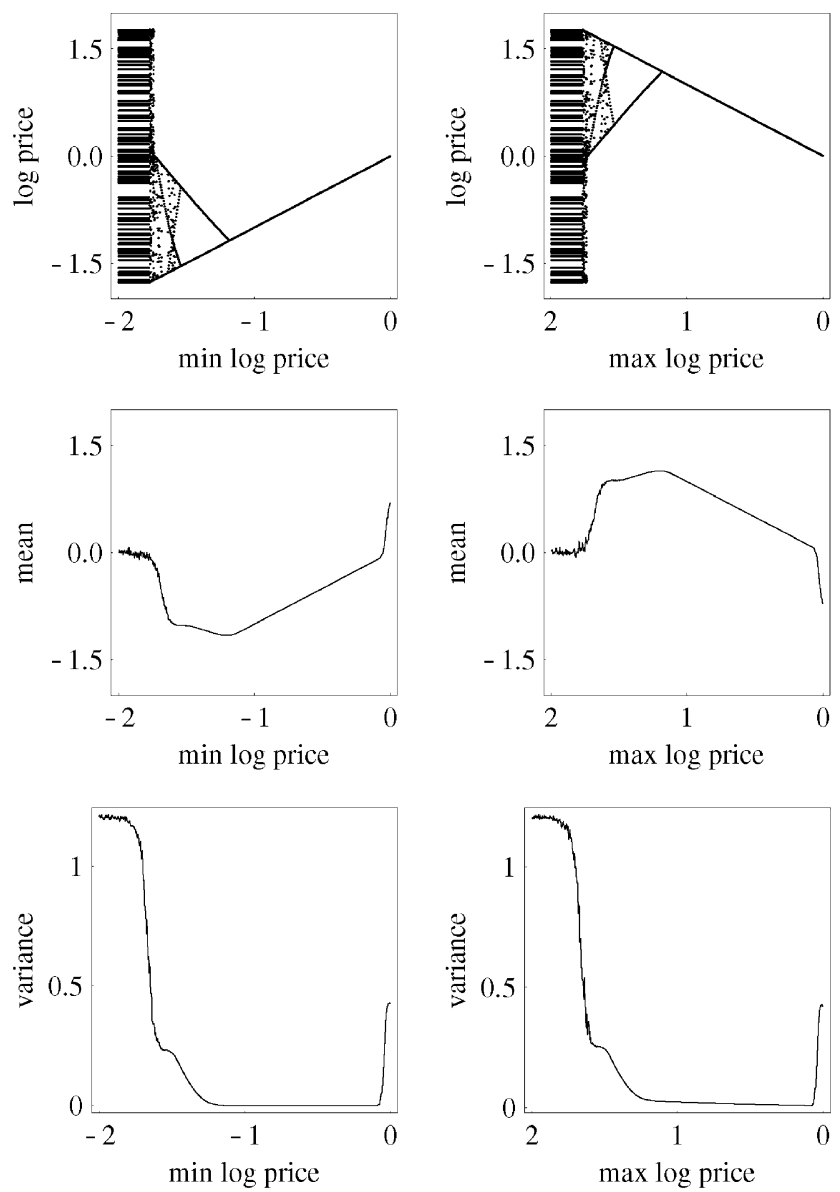

Fig. 5. The bifurcation diagrams in the first line of panels show how log prices react to more restrictive price boundaries. Log price limits are varied in 500 steps and $\log$ prices are plotted from $t=500-600$. The parameter setting is as in Fig. 4. The second and third lines of panels show the mean and the variance of the price process (buffeted with dynamic noise $N(0,0.1)$. All statistics are based on 10,000 observations.

To sum up, it turns out that the price dynamics under price limiters when both speculators react strongly is similar to the price behavior without imposing price limiters when either of the speculators react weakly. Consequently, one may conclude that the central authority eliminates the strong reactions of the speculators that push for the market to crash, leading to a bull market with high average price.

\section{Conditional price limiters}

Comparing the dynamics with and without price limiters, one can see that by imposing certain price limiters a central authority can effectively limit strong 
reactions of the speculators and stabilize the market price. However, such a policy may lead to substantial costs for the central authority. For example, to prevent the price from dropping below (going above) the minimum (maximum) price, the central authority permanently has to buy (sell) a fraction of the supplied (stored) commodity. Apart from the cost issue (e.g. for maintaining a buffer stock), nonnegative storage of the commodity prevents unlimited selling at high price limiters. To implement such interventions more successfully, we introduce a conditional price limiter mechanism in this section.

The top panel of Fig. 6 shows log price dynamics when the central authority switches between two price limiters: $S^{\min }=-1.3$ and $S^{\max }=1.3$. If the buffer stock exceeds a level of about \pm 15 a regime shift occurs. As can be seen, the price is thus stabilized either in the bull market or in the bear market. The duration of a regime is around 80 periods. Between the two regimes we observe a brief transient phase of around 20 periods in which the price evolves uncontrolled. Although the price is still distorted, conditional price limiters decrease the price volatility. The bottom panel of Fig. 6 presents the corresponding development of the buffer stock which now neither runs empty nor becomes infinitely large. Note that by buying low and selling high the interventions may be profitable in the long run.

It is quite interesting to see how the market prices are influenced by such a policy. Speculators may discover and respond to fixed price limiters. To prevent arbitrage opportunities, central authorities may thus use more flexible price limiters. Fig. 7 aims to demonstrate that the imposition of price limiters may create dramatic price changes such as bubbles and crashes. In the top panel of Fig. 7, it is assumed that the central authority stabilizes the log of the price in the bear market with a price limiter of -1.3 . However, from time-to-time interventions are briefly interrupted. For
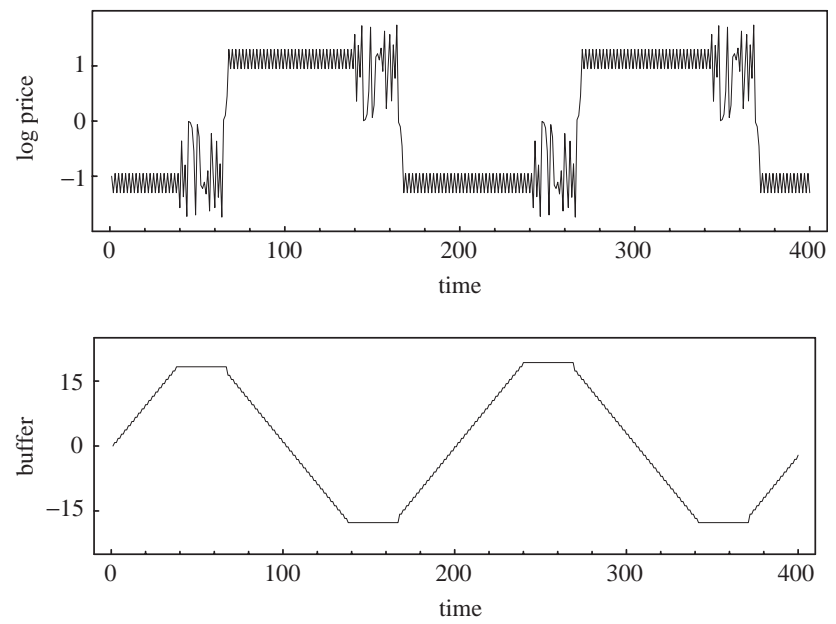

Fig. 6. The top panel shows log price dynamics when the central authority switches between log price limiters $S^{\min }=-1.3$ and $S^{\max }=1.3$. A change in regime occurs if the buffer stock exceeds a level of about \pm 15 . The bottom panel shows the corresponding evolution of the buffer stock. The other parameters are $a=1, b=4.5, c=1.5, d=1, m=1$ and $F=0$. 

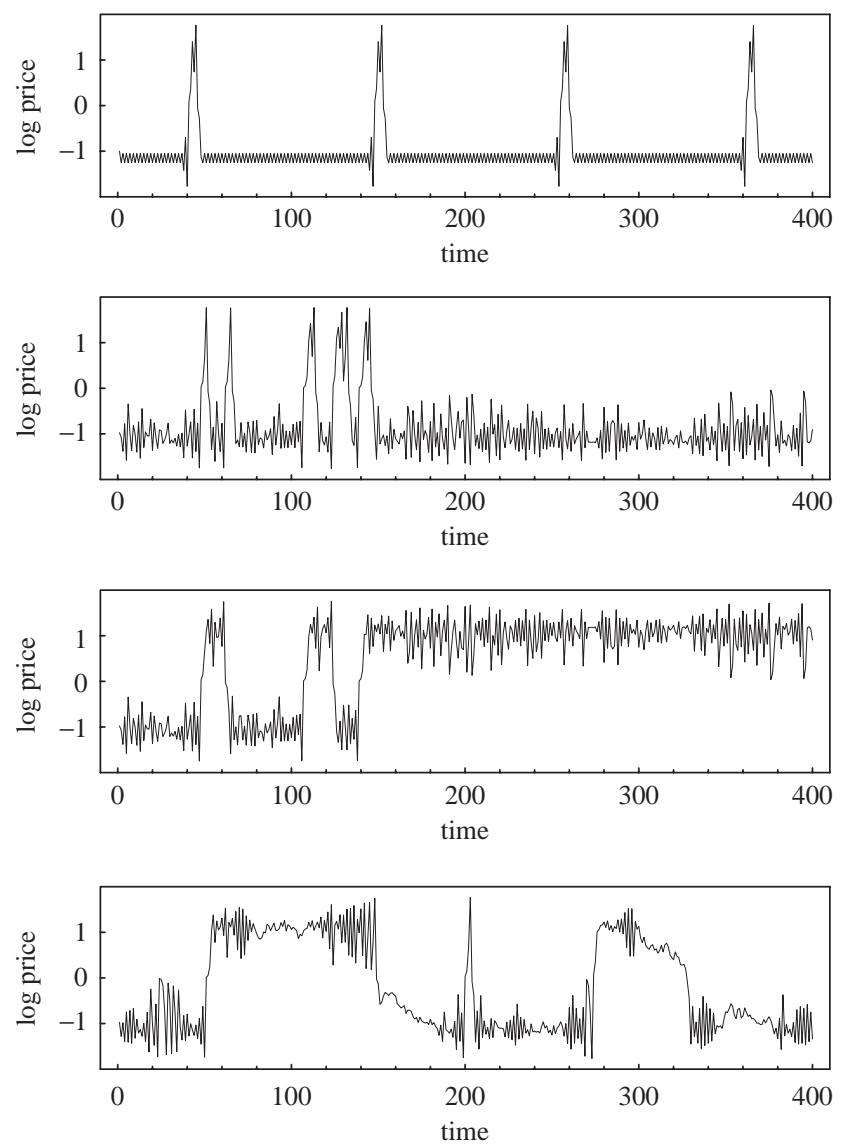

Fig. 7. Log price dynamics under different regimes. First panel: $S^{\min }=-1.25$, interrupted every 100 periods. Second panel: $S^{\min }=-1.3$, buffeted with dynamic noise $N(0,0.3)$. Third panel: $S^{\min }=-1.3$ and $S^{\max }=1.3$, both buffeted with dynamic noise $N(0,0.3)$. Fourth panel: Log price limiters as first-order autoregressive processes around \pm 1.3 with $\mathrm{AR}$ coefficients of 0.975 and noise $N(0,0.1)$. The other parameters are $a=1, b=4.5, c=1.5, d=1, m=1$ and $F=0$.

instance, the policy-makers may become afraid of the costs associated with their policy. But seeing that without interventions the price increases dramatically, the policy-makers may change their opinion once more and reactivate their old policy. Then the price is again bounded in the lower region. As a result, price patterns that resemble bubbles and crashes may simply emerge due to the activity of policymakers.

In the second panel of Fig. 7, the central authority applies a price limiter of $S^{\min }=-1.3$, buffeted with dynamic noise $N(0,0.3)$. Overall, the dynamics is still stabilized. However, now and then prices run away from bear markets. The reason is that the price limiter may be set too low to achieve the lock-in effect. In the third 
panel of Fig. 7, the central authority regulates the market with $S^{\text {min }}=-1.3$ and $S^{\max }=1.3$, both buffeted with dynamic noise $N(0,0.3)$. Again, temporary stabilizations either in the bull or in the bear market set in. ${ }^{5}$

Finally, in the fourth panel of Fig. 7, the price limiters are modeled as first-order auto-regressive processes around \pm 1.3 with AR coefficients of 0.975 and noise $N(0$, $0.1)$. Note that the price dynamics become increasingly realistic when the limiters are varied in a stochastic matter. Although the behaviors of consumers, producers and speculators are still deterministic, the price behavior is quite intricate. Visual inspection reveals bubbles and crashes, alternating periods of low and high volatility and also larger jumps, which may yield fat tail behavior of the distribution of the returns. ${ }^{6}$ What causes the increase in the complexity of the dynamics? The answer is quite simple. Fig. 5 already reveals that different price limiters result in different dynamic outcomes, e.g. a fixed point may be transformed into a limit cycle. In addition, perturbations of the price limiters work as shocks to the system and thus transient behavior may occur.

\section{Conclusions}

This paper is concerned with commodity price dynamics. Actual commodity prices fluctuate strongly: Not only is the price volatility high, but also severe bubbles and crashes regularly emerge. Hence, this topic is of great practical importance, particularly for the formulation of economic policy. Although producers and consumers are two primary participants in commodity markets, there are also other participants, such as speculators, who may have a marked effect both on the degree of price variability and on the success of any commodity price stabilization scheme.

Within our model, interactions between heterogeneous agents create complex bull and bear market fluctuations, which resemble the cyclical price dynamics of many commodity markets. Our model shows that: (i) the chartists are a source of market instability, as commonly believed; (ii) weak reaction of the speculators (either the fundamentalists or the chartists) can push the market to be either a bull or a bear market (through pitchfork bifurcations); and (iii) strong reaction of the speculators causes market prices to fluctuate irregularly between bull and bear markets (through homoclinic bifurcations). Furthermore, we investigate how price boundaries, which function identically to a recently suggested chaos control method, affect the price dynamics. We find that simple price limits (i) reduce the variability of prices quite strongly, (ii) are likely to shift the price in an adverse direction and (iii) may lead to an unsustainable buffer stock. The results are caused by a dynamic lock-in effect. By restricting the evolution of the price, the dynamics may become stuck in either the

\footnotetext{
${ }^{5}$ For a discussion of price caps and stochastic price processes see Dixit and Pindyck (1994).

${ }^{6}$ Indeed, applying the Hill tail index estimator procedure we find that the model is able to produce tail indices of around 3.5. Estimation of the Hurst coefficient for absolute returns reveals values of around 0.75 , indicating strong volatility clustering.
} 
bull or the bear market. However, jumping between bottoming and topping price limiters allows a central authority to manage the evolution of the buffer stock. Prices are then temporarily stabilized in the bull market or the bear market. But it should not be overlooked that whenever a central authority introduces a price stabilization scheme it changes the price discovery process. For instance, price limiters may trigger marked bubbles and crashes or volatility clustering.

The study of heterogeneous interacting agents has yielded a number of quite sophisticated models which have proven to be quite successful in explaining financial market dynamics. Our simple commodity market model is inspired by this approach and we would finally like to point out some interesting extensions. First of all, one may consider some other popular technical trading rules. For example, agents are often reported to extrapolate the most recent price trend. Moreover, as argued in Chiarella (1992) or Farmer and Joshi (2002), technical analysis may be nonlinear. Secondly, agents may involve some adaptive learning processes when choosing a particular trading strategy. For example, the behavior of chartists and fundamentalists may not be constant over time with respect to their reaction coefficients, and, although agents are boundedly rational, they may try to learn those coefficients. Alternatively, agents' expectations may follow some adaptive learning processes. Thirdly, agents may incorporate other switching mechanisms. As argued in Brock and Hommes (1998), one may assign each forecast rule a fitness function (which may depend on the historical performance of the rules) and then let the agent select a rule according to its fitness. Higher complexity may also be achieved by switching from a two-speculator type analysis to a real multi-agent market model (Lux and Marchesi, 2000). Of course, the behavior of both the producers and consumers may also be modeled in more detail. For instance, the producers may base their production decision on expected future prices and thus select between different kinds of forecast rules, as modeled in Brock and Hommes (1997). Finally, the working of different price limiter schemes may also be tested in a laboratory setting. Promising work on experimental asset pricing markets has been done by Smith (1991) or Sonnemans et al. (2004).

\section{Acknowledgements}

We thank two anonymous referees and the editor (Cars Hommes) for many valuable comments.

\section{Appendix A. Proof of Proposition 2}

The map $f(x)$ is not invertible if there exists an $\tilde{x}$ such that $f^{\prime}(\tilde{x})=0$. Let $z=1+d(x-F)^{2}$. It follows from (9) and $f^{\prime}(\tilde{x})=0$ that $\tilde{z}=1+d(\tilde{x}-F)^{2}$ satisfies

$$
(m+c-1) \tilde{z}^{2}+(b+c) \tilde{z}-2(b+c)=0 .
$$


We first assume that $m \geqslant 1$. Then $m+c>1$ and (A.1) has two real solutions

$$
\tilde{z}_{ \pm}=\frac{1}{2(m+c-1)}\left[-(b+c) \pm \sqrt{(b+c)^{2}+8(b+c)(m+c-1)}\right] .
$$

Note that $\tilde{x}$ exists only if $\tilde{z}>1$. Hence, $\tilde{z}_{+}$is the only possible choice. It can also be verified that $\tilde{z}>1$ is equivalent to $b>b_{2}=m-1 \geqslant 0$. Therefore, when $m \geqslant 1$, the map is invertible for $b \leqslant b_{2}$ and not invertible for $b>b_{2}$.

We now assume that $m<1$. If $m+c=1$, then $\tilde{z}=2$. If $m+c>1$, then $\tilde{z}_{+}>1$. Thus, the map is not invertible for $m+c \geqslant 1$. If $m+c<1$, then $\tilde{z}$ is positive only if $b>-8(m-1)-7 c$. Also, $\tilde{z}>1$ leads to $b<m-1$, which is impossible. Hence, the map is invertible. This completes the proof.

\section{References}

Brennan, M., 1958. The supply of storage. American Economic Review 48, 50-72.

Brock, W., Hommes, C., 1997. A rational route to randomness. Econometrica 65, 1059-1095.

Brock, W., Hommes, C., 1998. Heterogeneous beliefs and routes to chaos in a simple asset pricing model. Journal of Economic Dynamics Control 22, 1235-1274.

Canoles, B., Thompson, S., Irwin, S., France, V., 1998. An analysis of the profiles and motivations of habitual commodity speculators. Journal of Futures Markets 18, 765-801.

Chambers, M., Bailey, R., 1996. A theory of commodity price fluctuations. Journal of Political Economy 104, 924-957.

Chiarella, C., 1992. The dynamics of speculative behavior. Annals of Operations Research 37, 101-123.

Chiarella, C., He, X.-Z., 2001. Asset price and wealth dynamics under heterogeneous expectations. Quantitative Finance 1, 509-526.

Chiarella, C., He, X.-Z., 2003. Dynamics of beliefs and learning under $a_{-} L$ processes - the heterogeneous case. Journal of Economic Dynamics and Control 27, 503-531.

Chiarella, C., Dieci, R., Gardini, L., 2002. Speculative behavior and complex asset price dynamics. Journal of Economic Behavior and Organization 49, 173-197.

Corron, N., Pethel, S., Hopper, B., 2000. Controlling chaos with simple limiters. Physical Review Letters $84,3835-3838$

Day, R., Huang, W., 1990. Bulls, bears and market sheep. Journal of Economic Behavior and Organization 14, 299-329.

Deaton, A., Laroque, G., 1992. On the behavior of commodity prices. Review of Economic Studies 59, $1-23$.

Deaton, A., Laroque, G., 1996. Competitive storage and commodity price dynamics. Journal of Political Economy 104, 896-923.

Devaney, R., 1989. An Introduction to Chaotic Dynamical Systems, second ed. Addison-Wesley, Reading, MA.

Dieci, R., Bischi, G.I., Gardini, L., 2001. From bi-stability to chaotic oscillations in a macroeconomic model. Chaos, Solitons and Fractals 12, 805-822.

Dixit, A., Pindyck, R., 1994. Investment Under Uncertainty. Princeton University Press, Princeton.

Draper, D., 1985. The small public trader in futures markets. In: Peck, A. (Ed.), Futures Markets: Regulatory Issues. American Enterprise Institute for Public Policy Research, Washington, pp. 211-269.

Farmer, D., Joshi, S., 2002. The price dynamics of common trading strategies. Journal of Economic Behavior and Organization 49, 149-171.

Goeree, J., Hommes, C., 2000. Heterogeneous beliefs and the non-linear cobweb model. Journal of Economic Dynamics and Control 24, 761-798. 
Hommes, C., 1994. Dynamics of the cobweb model with adaptive expectations and nonlinear supply and demand. Journal of Economic Behavior and Organization 24, 315-335.

Hommes, C., 2001. Financial markets as nonlinear adaptive evolutionary systems. Quantitative Finance 1, $149-167$.

Kaas, L., 1998. Stabilizing chaos in a dynamical macroeconomic model. Journal of Economic Behavior and Organization 33, 313-332.

Kirman, A., 1991. Epidemics of opinion and speculative bubbles in financial markets. In: Taylor, M. (Ed.), Money and Financial Markets. Blackwell, Oxford, pp. 354-368.

Kopel, M., 1997. Improving the performance of an economic system: controlling chaos. Journal of Evolutionary Economics 7, 269-289.

Lux, T., Marchesi, M., 2000. Volatility clustering in financial markets: a micro-simulation of interacting agents. International Journal of Theoretical and Applied Finance 3, 675-702.

Newbery, D., Stiglitz, J., 1981. The Theory of Commodity Price Stabilization: a Study in the Economics of Risk. Clarendon Press, Oxford.

Osborne, T., 2003. Market news in commodity price theory: applications to the Ethiopian grain market. Review of Economic Studies, in press.

Ott, E., Grebogi, C., Yorke, J., 1990. Controlling chaos. Physical Review Letters 64, 1196-1199.

Parthasarathy, S., Sinha, S., 1995. Controlling chaos in unidimensional maps using constant feedback. Physical Review E 51, 6239-6242.

Pyragas, K., 1992. Continuous control of chaos by self-controlling feedback. Physical Letters A 170 , $421-428$.

Sanders, D., Irwin, S., Leuthold, R., 2000. Noise trader sentiment in futures markets. In: Goss, B. (Ed.), Models of Futures Markets. Routledge, London, pp. 86-116.

Smidt, S., 1965. Amateur speculators: a survey of trading strategies, information sources and patterns of entry and exit from commodity futures markets by non-professional speculators. Cornell Studies in Policy and Administration, Cornell University.

Smith, V., 1991. Papers in Experimental Economics. Cambridge University Press, Cambridge.

Sonnemans, J., Hommes, C., Tuinstra, J., van de Velden, H., 2004. The instability of a heterogeneous cobweb economy: a strategy experiment on expectation formation. Journal of Economic Behavior and Organization 54, 453-481.

Stoop, R., Wagner, C., 2003. Scaling properties of simple limiter control. Physical Review Letters 90, 154101-154111.

Wagner, C., Stoop, R., 2000. Optimized chaos control with simple limiters. Physical Review E 63, 154101.1-154101.4.

Waugh, F., 1944. Does the consumer benefit from price instability? Quarterly Journal of Economics 58, 602-614.

Weiner, R., 2002. Sheep in wolves' clothing? Speculators and price volatility in petroleum futures. Quarterly Review of Economics and Finance 42, 391-400.

Westerhoff, F., 2003. Speculative markets and the effectiveness of price limits. Journal of Economic Dynamics and Control 28, 493-508.

Westerhoff, F., Wieland, C., 2004. Exchange rate dynamics, central bank interventions and chaos control methods. Journal of Economic Behavior and Organization, in press.

Williams, J., Wright, B., 1991. Storage and Commodity Markets. Cambridge University Press, Cambridge. 